\title{
Expansion wave and cavitation bubble generation by underwater shock wave reflection from the interface
}

\author{
Kiyonobu OHTANI* and Toshihiro OGAWA* \\ *Institute of Fluid Science, Tohoku University \\ 2-1-1 Katahira, Aoba, Sendai 980-8577, Japan \\ E-mail: ohtani@ifs.tohoku.ac.jp
}

Received 9 May 2016

\begin{abstract}
This paper reports an experimental result of expansion wave and cavitation bubble generation by underwater shock wave reflection at the interface for understanding of shock wave interaction phenomena related to the mechanism of shock wave human tissue damage. Underwater shock wave was generated by detonating a micro-explosive (silver azide pellet). The process of underwater shock wave reflection at the interface, expansion wave and cavitation bubble generation were visualized by shadowgraph method and recorded by an ultra-high-speed framing camera at high spatiotemporal resolution. The pressure time history near the interface in water was measured simultaneously by a needle hydrophone. High-speed shadowgraph sequential images show that a compression wave (shock wave) was reflected from a thin interface plate (Polystyrene, acrylic, Teflon, thickness of $t=0.5 \mathrm{~mm}$ ), and expansion wave was then generated in water by reflection from the air. In addition, a cavitation bubble was created behind the expansion wave. The simultaneously measured pressure history also shows that an expansion wave propagated behind shock wave. The negative peak pressure of expansion wave in the case of a thin plate shock wave interaction was larger than that in water surface interaction.
\end{abstract}

Key words : Shock wave, Expansion wave, Acoustic impedance, Shadowgraph, Pressure history

\section{Introduction}

Underwater shock wave and expansion wave propagation and their induced phenomena such a cavitation bubble generation, related to human tissue damage by shock wave interacting with human body, are an important area of research for shock wave medical and biomedical application (Delius 1994, Tomita 1994, Takayama and Saito 2004, Nakagawa et al., 2011 and Ohtani and Ogawa 2015). Human tissue damage is caused by following effects of pressure wave propagation, that is, a rapid pressure increase and high overpressure interacting by shock wave, and drastic pressure decrease and cavitation bubble generation by expansion wave. Kedrinskii $(1975,1976)$ and Ohtani et al., $(2009,2011)$ have investigated the expansion wave generation by small charge-induced underwater shock wave reflecting from the water surface. Cavitation bubble was generated at the area under the water surface by interacting the expansion wave. Acoustic impedance $Z$ is defined as $Z=\rho c$, where $\rho$ is the density of the material, $c$ is the speed of sound. Additionally, the reflection coefficient $R_{p}$ and the transmission coefficient $T_{p}$ are determined by the following equations,

$$
\begin{aligned}
& R_{p}=\frac{P_{r}}{P_{i}}=\frac{Z_{2}-Z_{1}}{Z_{2}+Z_{1}} \\
& T_{p}=\frac{P_{t}}{P_{i}}=\frac{2 Z_{2}}{Z_{2}+Z_{1}}
\end{aligned}
$$

where $P_{i}, P_{r}$ and $P_{t}$ are respectively, the incident, reflected and transmitted shock wave peak pressure. $Z_{1}$ and $Z_{2}$ 
are the acoustic impedance of the material on the incident and transmission sides, respectively. Therefore, in the case of the interface of water and air, $R_{p}$ is a negative value, that is, reflective pressure wave become expansion wave.

Human body is composed of various tissues has different acoustic impedance. The acoustic impedance of human tissue (Nakagawa et al., 2011) ranges from hard tissue (for example, bone $\left(Z=2.1-7.8 \times 10^{6} \mathrm{~kg} /\left(\mathrm{m}^{2} \mathrm{~s}\right)\right)$ ) to soft tissue (brain $\left(Z=1.58 \times 10^{6} \mathrm{~kg} /\left(\mathrm{m}^{2} \mathrm{~s}\right)\right)$ ) and biological liquid (blood $\left(Z=1.61 \times 10^{6} \mathrm{~kg} /\left(\mathrm{m}^{2} \mathrm{~s}\right)\right)$ ). Naturally, there is individual variability in acoustic impedance. Shock wave propagation phenomena in human tissue is very complex by the different acoustic impedance, and the position of each tissues

The effect of acoustic impedance is considered to be important in shock wave interaction phenomena related to shock wave human tissue damage. According to the previous research about underwater shock wave interaction phenomena with a $1.0 \mathrm{~mm}$ thickness plate (Ohtani and Ogawa 2015), an expansion wave was generated by the transmitted shock wave reflecting from the interface of an acrylic and aluminum thin plate and air, with a cavitation bubble subsequently occurring near the interface. The negative peak pressure of the expansion wave in the case of water, acrylic plate and air interface was lower than in the case of water and air, because of the large difference in acoustic impedance between the propagation medium and air. However, it is not still enough for understanding of the shock wave interaction phenomena related to the shock wave human tissue damage.

In this study, an experiment of expansion wave and cavitation bubble generation by an underwater shock wave reflection with the interface was performed for understanding of shock wave interaction phenomena related to the mechanism of shock wave human tissue damage. Especially, the effect of plate thickness and acoustic impedance for the shock wave interaction with the interface was investigated. Actually, shock wave propagation in human tissue is complex three-dimensional phenomena. However, in this study, we have investigated shock wave interaction phenomena in a simple 1-dimension model by a thin plate. The process of reflected shock wave and expansion wave propagation by micro-explosive induced underwater shock wave interaction with the interface of water, a thin plate and air was visualized by shadowgraph method and recorded by ultra-high-speed framing camera at high spatiotemporal resolution. The pressure time history near the interface in water was measured simultaneously with the ultra-high-speed shadowgraph optical visualization by a pressure sensor.

\section{Experimental setup}

Figure 1 shows the experimental setup for observation of expansion wave and cavitation bubble generation by an underwater shock wave reflecting the interface in a stainless steel water-filled chamber. The water chamber with dimensions of $200 \times 200 \times 200 \mathrm{~mm}$ was used, which was equipped with a $200 \times 200 \mathrm{~mm}$ acrylic observation window on the both side of the water chamber. An underwater shock wave was generated by detonating a micro-explosive pellet (silver azide, $\mathrm{AgN}_{3}$, Showa Kinzoku Kogyo Co., Ltd., density of $3.8 \mathrm{~g} / \mathrm{cm}^{3}$, weight of $10 \pm 0.1 \mathrm{mg}$, diameter of $1.5 \mathrm{~mm}$, length of $1.5 \mathrm{~mm}$ ). The micro-explosive pellet is glued at the tip of a quartz optical fiber (G.C. 600/750, Fujikura Ltd.) and placed at a distance of about $20 \mathrm{~mm}$ from the interface, that is the interface of water and a thin plate. In this study, a thin plate made of acrylic, polystyrene (PS), and Teflon (TF) $(100 \times 100 \mathrm{~mm}$, thickness of $t=0.5,1.0$, and $2.0 \mathrm{~mm})$ are used as the interface with water.

A Q-switched pulsed Nd: YAG laser beam (SAGA220, B.M. Industries, $1064 \mathrm{~nm}$ in wavelength, $7 \mathrm{~ns}$ pulse

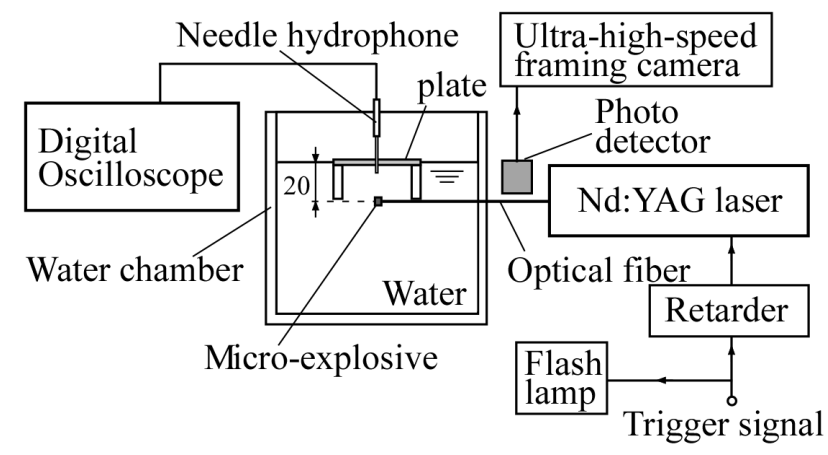

Fig. 1 Schematic diagram of the experimental setup for observation of expansion wave and cavitation bubble generation near the interface. 
Table 1 List of acoustic impedance values.

\begin{tabular}{c|c|c|c}
\hline \hline Material & $\begin{array}{c}\text { Density } \\
\rho\left[\mathrm{kg} / \mathrm{m}^{3}\right]\end{array}$ & $\begin{array}{c}\text { Sound of speed } \\
c[\mathrm{~m} / \mathrm{s}]\end{array}$ & $\begin{array}{c}\text { Acoustic impedance } \\
Z=\rho c\left[\mathrm{~kg} /\left(\mathrm{m}^{2} \mathrm{~s}\right)\right]\end{array}$ \\
\hline Air $^{* 1}$ & 1.2929 & 331.45 & 428.6 \\
Water $^{* 1}$ & $1.00 \times 10^{3}$ & 1500 & $1.50 \times 10^{6}$ \\
Polystyrene $^{* 2}$ & $1.048 \times 10^{3}$ & 2266 & $2.37 \times 10^{6}$ \\
Acrylic $^{* 2}$ & $1.184 \times 10^{3}$ & 2640 & $3.13 \times 10^{6}$ \\
Teflon $^{2}$ & $2.15 \times 10^{3}$ & 1390 & $2.99 \times 10^{6}$ \\
\hline
\end{tabular}

${ }^{* 1}$ National Astronomical Observatory of Japan (2015)

${ }^{* 2}$ Raum and Brandt (2003)

duration, about $25 \mathrm{~mJ}$ per pulse) was guided through the optical fiber and then ignited the micro-explosive pellet. Table 1 shows the list of acoustic impedance values for air, water (National Astronomical Observatory 2015) and solid materials (Raum and Brandt 2003) used in this study. The acoustic impedance of human tissue is less than about $8.0 \mathrm{x}$ $10^{6} \mathrm{~kg} /\left(\mathrm{m}^{2} \mathrm{~s}\right)$ (Nakagawa et al. 2011). A human tissue is able to simulate about the acoustic impedance by using these materials.

The process of expansion wave and cavitation bubble generation were visualized at ultra-high-speed by the shadowgraph method and recorded by the ultra-high-speed framing camera (Imacon200, DRS Technologies Inc., resolution of $1200 \times 980$ pixel per frame). The optical setup for the shadowgraph method was constructed though use of the parallel beam collimating flash lamp light (PE-5651, Panasonic Inc.) and a parabolic mirror (focal length of 1,000 $\mathrm{mm}$, diameter of $190 \mathrm{~mm}$ ) (Ohtani and Ogawa 2015). The observation was performed at the inter-frame time of $500 \mathrm{~ns}$ (2Mfps) and the exposure time of $10 \mathrm{~ns}$.

The pressure history in water near the interface was measured simultaneously with the ultra-high-speed shadowgraph optical visualization by a spatiotemporal needle hydrophone (Platte Needle Probe, Müller Instruments, detectable pressure range of -10 to $200 \mathrm{MPa}$, sensitive diameter of $0.5 \mathrm{~mm}$, rise time of $50 \mathrm{~ns}$ ) and recorded by a digital oscilloscope (DL750, YOKOGAWA Corp., sampling speed of 10MS/s). The needle hydrophone was placed under a thin plate in water though the plate with a hole at its center.

\section{Results and discussions}

\subsection{High-speed shadowgraph sequential images}

Figure 2-5 show high-speed shadowgraph sequential images of underwater shock wave reflection the interface. The case of water-air interface is shown in Fig. 2. At $12.0 \mu \mathrm{s}$ after ignition of the micro explosive pellet, the induced underwater shock wave almost reached the interface. The shock wave was reflected from the interface of water and air as an expansion wave. The pressure near the water surface was decreased by propagation of the expansion wave, and cavitation bubble was created behind the expansion wave. Especially, the area near the water surface became dark by creating of cavitation bubble in large numbers.

The case of water, polystyrene (PS) (plate thickness of $t=0.5 \mathrm{~mm}$ ) and air interface is shown in Fig. 3. The underwater shock wave was reflected at the interface, along with multiple other waves. The first reflected wave is a reflected shock wave (compressive wave). The secondary wave is an expansion wave, because of cavitation bubble is created behind this wave. The first wave is reflected form the thin PS plate, and the secondary wave is the reflected expansion wave from the interface of PS and air. This expansion wave and the subsequent waves were generated by the transmitted compressive wave propagating and reflecting repeatedly in the thin PS plate. The interval between the first and the secondary reflected wave fronts measured by the sequential images was about $0.73 \mathrm{~mm}$, equal to the propagation distance in water over the time required for propagating across the thin plate at the sound speed of PS.

The case of water, acrylic (plate thickness of $t=0.5 \mathrm{~mm}$ ) and air interface is shown in Fig. 4. Acrylic has higher acoustic impedance than PS. In this case, the underwater shock wave was also reflected at the interface, followed by multiple other reflected waves. In the same way as in the case of a PS plate, the first reflected wave is a shock wave and the secondary wave is an expansion wave, behind this wave cavitation bubble is created. But the wave front of the first reflected wave became clear, that is, the shock wave pressure was increased by reflection with the high acoustic impedance medium relative to PS. The interval between the first and the second reflected wave front is shorter than that in the case of a PS plate.

The case of water, Teflon (TF) (plate thickness of $t=0.5 \mathrm{~mm}$ ) and air interface is shown in Fig. 5 . TF has acoustic 

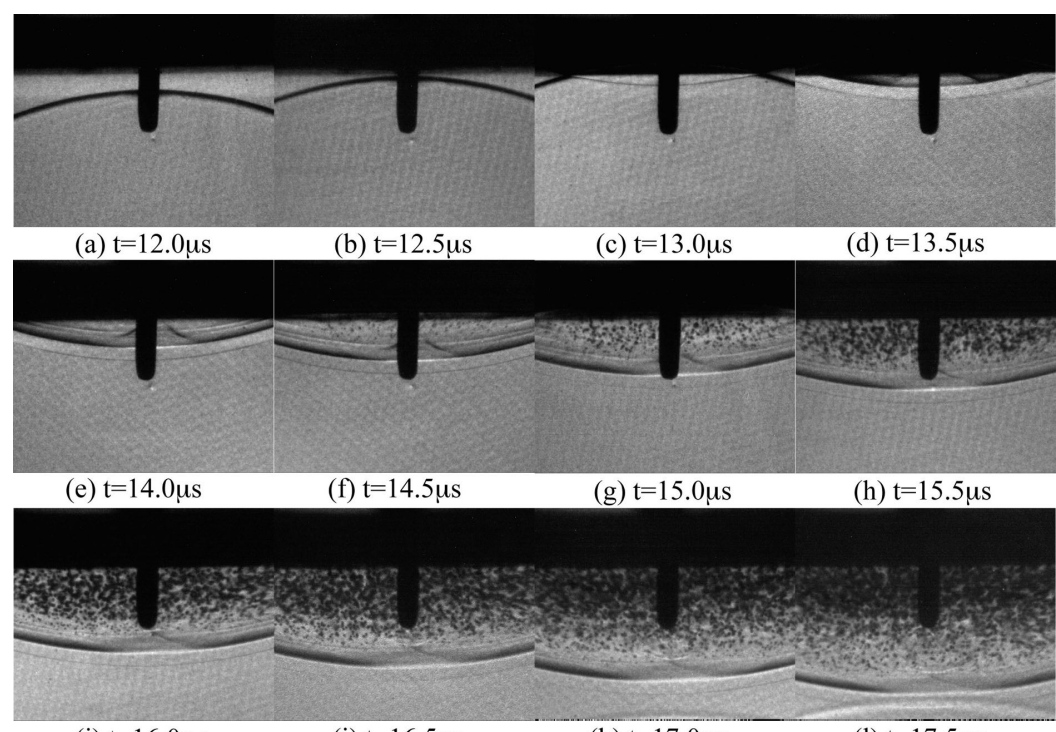
(i) $\mathrm{t}=16.0 \mu \mathrm{s}$
(j) $\mathrm{t}=16.5 \mu \mathrm{s}$
(k) $\mathrm{t}=17.0 \mu \mathrm{s}$
(1) $t=17.5 \mu \mathrm{s}$

Fig. 2 Sequential shadowgraph images of reflected shock wave and expansion wave propagation by underwater shock wave interaction with the interface of water and air.

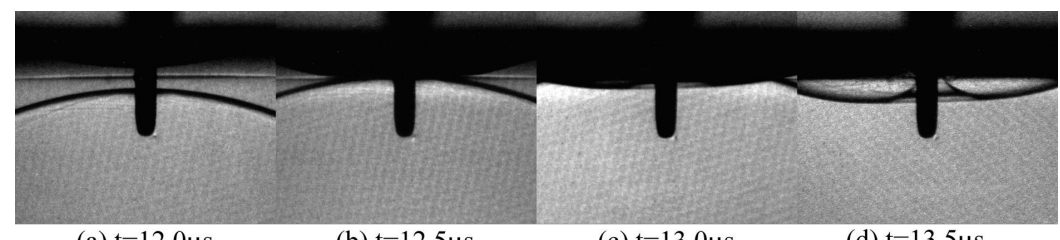

(a) $\mathrm{t}=12.0 \mu \mathrm{s}$

(b) $\mathrm{t}=12.5 \mu \mathrm{s}$

(c) $\mathrm{t}=13.0 \mu \mathrm{s}$

(d) $\mathrm{t}=13.5 \mu \mathrm{s}$

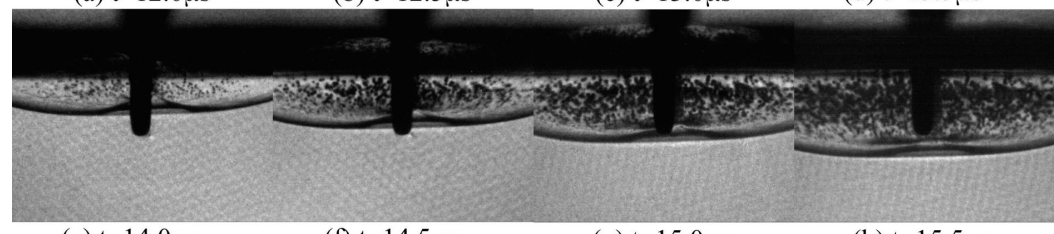

(e) $\mathrm{t}=14.0 \mu \mathrm{s}$

(f) $\mathrm{t}=14.5 \mu \mathrm{s}$

(g) $\mathrm{t}=15.0 \mu \mathrm{s}$

(h) $\mathrm{t}=15.5 \mu \mathrm{s}$

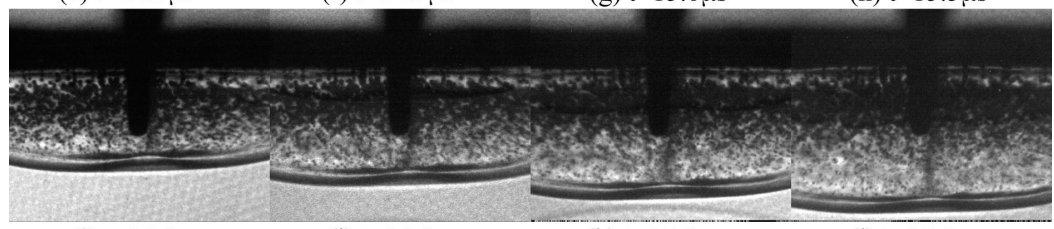

$\begin{array}{llll}\text { (i) } \mathrm{t}=16.0 \mu \mathrm{s} & \text { (j) } \mathrm{t}=16.5 \mu \mathrm{s} & \text { (k) } \mathrm{t}=17.0 \mu \mathrm{s} & \text { (1) } \mathrm{t}=17.5 \mu \mathrm{s}\end{array}$

Fig. 3 Sequential shadowgraph images of reflected shock wave and expansion wave propagation by underwater shock wave interaction with the interface of water, polystyrene (PS) and air. (plate thickness of $0.5 \mathrm{~mm}$ )

impedance that is higher than PS, but is lower than acrylic, and slower sound speed than PS and acrylic. The interval between the first and the second reflected wave front is longer than that in the case of a PS and acrylic plate, because of lower sound speed of Teflon.

These shock wave propagation behaviors in the case of plate thickness of $0.5 \mathrm{~mm}$ agree with the result in plate thickness of $1.0 \mathrm{~mm}$ in the previous study (Ohtani and Ogawa 2015).

\subsection{Pressure histories near the interface}

Figure 6 shows the measured pressure histories near the interface in water at a distance of about $3 \mathrm{~mm}$ from the interface simultaneously with the ultra-high-speed shadowgraph optical visualization by a needle hydrophone.

In all interface cases, at about 10-11 us after ignition of the micro-explosive pellet, the incident shock wave reached the tip of the needle hydrophone, after which the pressure raised sharply and reduced immediately. Further, after $14 \mu \mathrm{s}$, the reflected wave was detected. In the interface case of water and air, as shown in Fig. 6(a), when the 


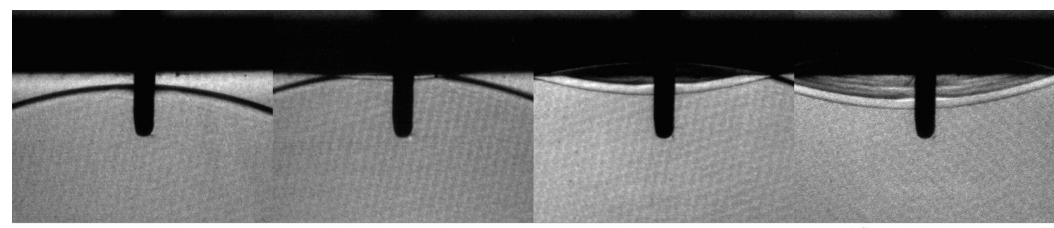

(a) $\mathrm{t}=12.0 \mu \mathrm{s}$ (b) $\mathrm{t}=12.5 \mu \mathrm{s}$ (c) $\mathrm{t}=13.0 \mathrm{us}$ (d) $\mathrm{t}=13.5 \mu \mathrm{s}$
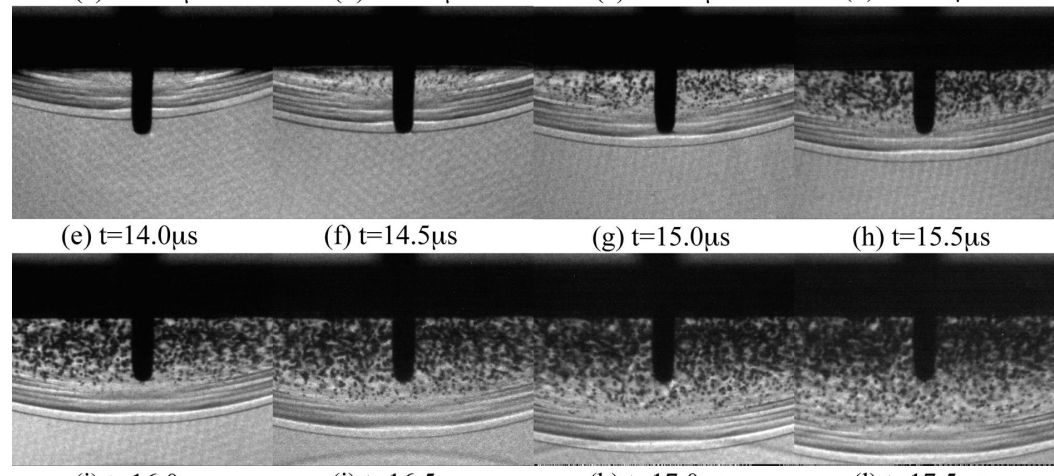
(i) $\mathrm{t}=16.0 \mathrm{us}$
(j) $\mathrm{t}=16.5 \mu \mathrm{s}$
(k) $\mathrm{t}=17.0 \mathrm{us}$
(1) $\mathrm{t}=17.5 \mu \mathrm{s}$

Fig. 4 Sequential shadowgraph images of reflected shock wave and expansion wave propagation by underwater shock wave interaction with the interface of water, acrylic and air. (plate thickness of $0.5 \mathrm{~mm}$ )

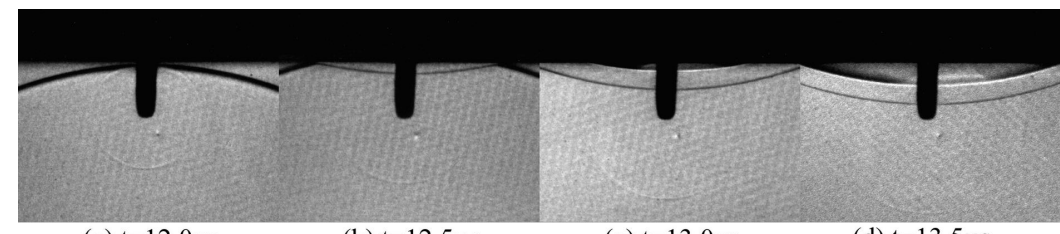

(a) $\mathrm{t}=12.0 \mathrm{us}$

(b) $t=12.5 \mu \mathrm{s}$

(c) $\mathrm{t}=13.0 \mathrm{us}$

(d) $\mathrm{t}=13.5 \mu \mathrm{s}$

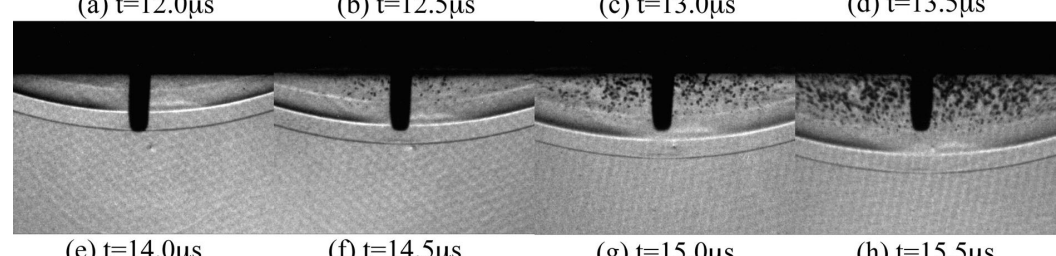

(e) $\mathrm{t}=14.0 \mu \mathrm{s}$

(f) $\mathrm{t}=14.5 \mu \mathrm{s}$

(g) $\mathrm{t}=15.0 \mathrm{us}$

(h) $\mathrm{t}=15.5 \mathrm{us}$

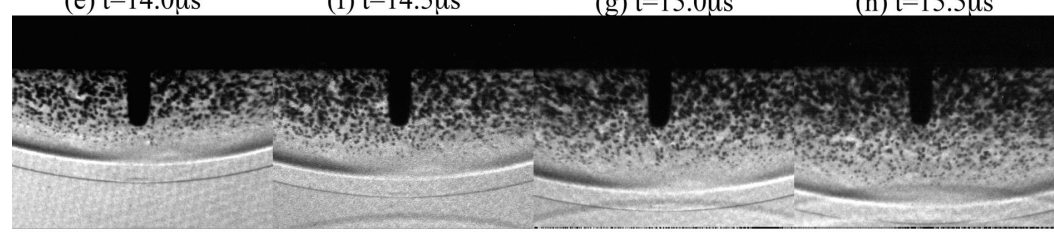

(i) $\mathrm{t}=16.0 \mu \mathrm{s}$

(j) $\mathrm{t}=16.5 \mu \mathrm{s}$

(k) $t=17.0 \mu \mathrm{s}$

(1) $\mathrm{t}=17.5 \mu \mathrm{s}$

Fig. 5 Sequential shadowgraph images of reflected shock wave and expansion wave propagation by underwater shock wave interaction with the interface of water, Teflon (TF) and air. (plate thickness of $0.5 \mathrm{~mm}$ )

reflected wave, that is, the expansion wave, arrived, the pressure decreased to about $-4.6 \mathrm{MPa}$. However, in the case of water of water, a polystyrene (PS) thin plate and air as shown in Fig. 6(b-1, b-2, b-3), two reflected waves with relatively high amplitude arrived as a shock wave and an expansion wave compare to the interface case of water and air. The negative peak pressure of the expansion wave reached -9.9 MPa (1.0 mm PS plate), which is lower than the interface case of water and air. But in the case of a thick plate comparatively (thickness $t=2.0 \mathrm{~mm}$ ), the wave profile of reflected shock wave was distorted, and the pressure pulse width was increased by the effect of plate thickness. In the case of water, a thin acrylic plate and air as shown in Fig. 6(c-1, c-2, c-3), two reflected waves arrived as a shock wave and an expansion wave as is the case with a PS thin plate. The peak overpressure of the reflected shock wave was lager amplitude than that in the case of a PS thin plate, because of the reflection coefficient $R_{p}$ is lager. In the case of water, a thin Teflon (TF) plate and air as shown in Fig. 6(d-1, d-2, d-3), similarly, two reflected waves arrived as a shock wave and an expansion wave. In a TF thin plate case at $1.0 \mathrm{~mm}$ thickness, the wave profile of reflected shock wave was distorted, and the pressure pulse width was increased. 


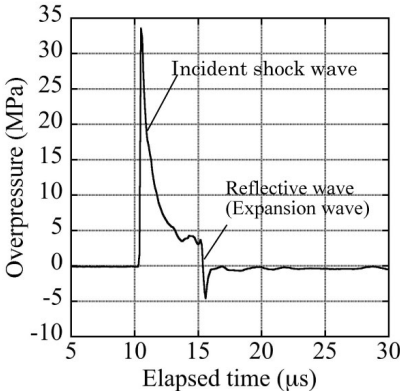

(a) water, $h=3.0 \mathrm{~mm}$

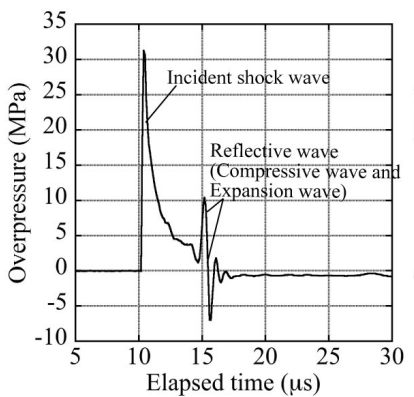

(c-1) Acrylic, $t=0.5 \mathrm{~mm}, h=3.2 \mathrm{~mm}$

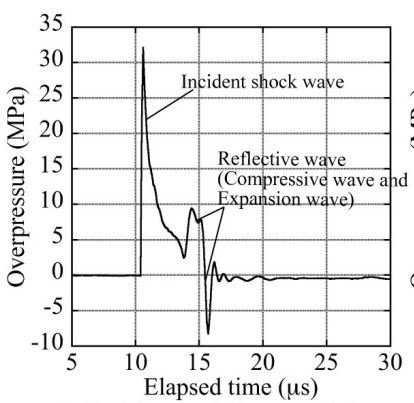

(d-2) Teflon, $t=1.0 \mathrm{~mm}, h=2.6 \mathrm{~mm}$

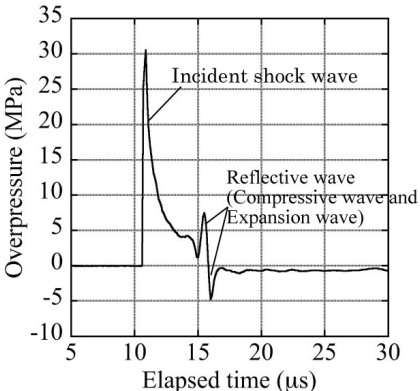

(b-1) PS, $t=0.5 \mathrm{~mm}, h=3.2 \mathrm{~mm}$

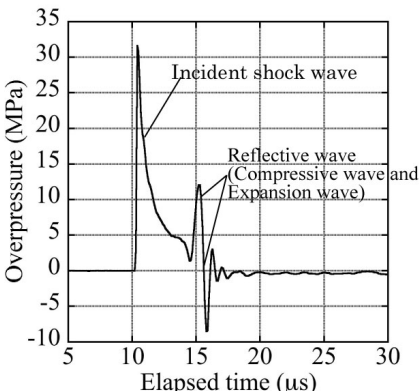

(c-2) Acrylic, $t=1.0 \mathrm{~mm}, h=3.2 \mathrm{~mm}$

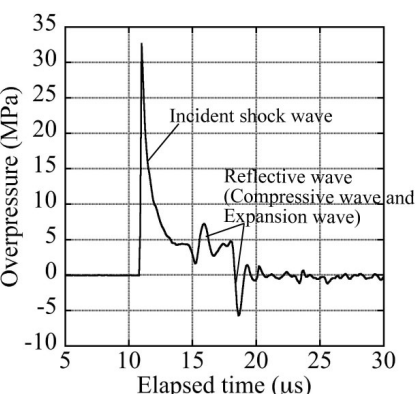

(d-3) Teflon, $t=2.0 \mathrm{~mm}, h=3.2 \mathrm{~mm}$

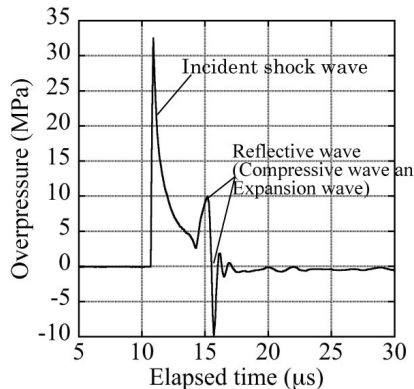

(b-2) PS, $t=1.0 \mathrm{~mm}, h=3.1 \mathrm{~mm}$

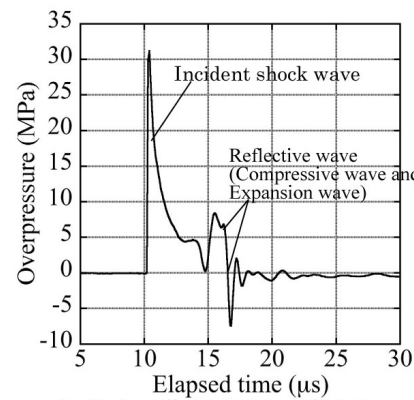

(c-3) Acrylic, $t=2.0 \mathrm{~mm}, h=3.2 \mathrm{~mm}$

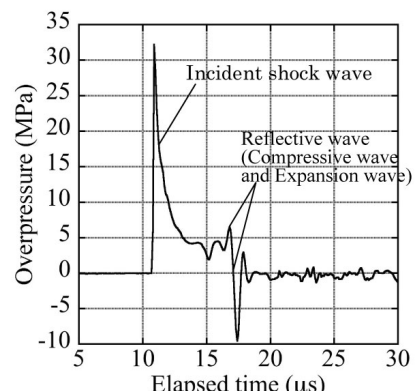

(b-3) PS, $t=2.0 \mathrm{~mm}, h=3.2 \mathrm{~mm}$

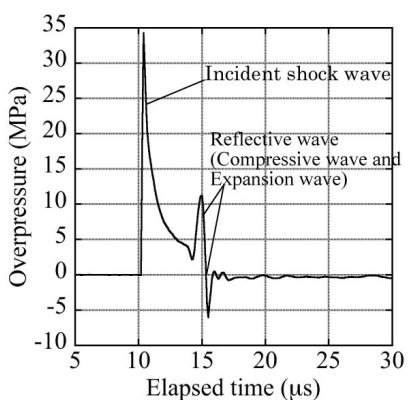

(d-1) Teflon, $t=0.5 \mathrm{~mm}, h=3.0 \mathrm{~mm}$

Fig. 6 Pressure histories near the interface of underwater shock wave interaction with the interface.

Table 2 List of reflection coefficients.

\begin{tabular}{|c|c|c|c|}
\hline \multicolumn{2}{|c|}{ Interface / plate thickness (mm) } & $R_{p-\exp }=P_{r} / P_{i}$ & $R_{p-\text { theo }}=(Z 2-Z 1) /(Z 2+Z 1)$ \\
\hline \multirow{3}{*}{ water-PS-air } & 0.5 & 0.24 & \multirow{3}{*}{0.21} \\
\hline & 1.0 & 0.30 & \\
\hline & 2.0 & 0.20 & \\
\hline \multirow{3}{*}{ water-acrylic-air } & 0.5 & 0.33 & \multirow{3}{*}{0.35} \\
\hline & 1.0 & 0.38 & \\
\hline & 2.0 & 0.26 & \\
\hline \multirow{3}{*}{ water-Teflon-air } & 0.5 & 0.33 & \multirow{3}{*}{0.33} \\
\hline & 1.0 & 0.29 & \\
\hline & 2.0 & 0.22 & \\
\hline
\end{tabular}

In all interface cases, in term of the negative peak pressure of the expansion wave, the negative peak pressure of a thin plate and air is lower than at water surface within the range of this experimental condition. But, in the negative peak pressure these experimental results show no clear trend with the different plate thickness.

Table 2 summarizes the data for reflection coefficients $R_{p}$ calculated by using Eq. (1) from the measured shock peak over pressure data and acoustic impedance values (Table 1). The reflection coefficient calculated form the experimental results $R_{p \text {-exp }}$ at a plate thickness of $t=1.0 \mathrm{~mm}$ is larger than at $t=0.5 \mathrm{~mm}$ in the plate case of PS and acrylic. However, $R_{p \text {-exp }}$ is smaller with the thicker plate by the distorted pressure wave profile. The theoretically calculated reflection coefficient $R_{p \text {-theo }}$ is in rough agreement with $R_{p-\exp }$. 


\section{Conclusion}

Expansion wave and cavitation bubble generation by an underwater shock wave reflecting at the interface for understanding of shock wave interaction phenomena related to the mechanism of shock wave human tissue damage was experimentally investigated. A compression wave (shock wave) was reflected from a thin interface plate (PS, acrylic, Teflon, thickness of $t=0.5 \mathrm{~mm}, 1.0 \mathrm{~mm}$ and $2.0 \mathrm{~mm}$ ), and expansion wave was then generated in water by reflection from the air. In addition, a cavitation bubble was created behind the expansion wave. The simultaneously measured pressure history also shows that an expansion wave propagated behind shock wave. The negative peak pressure of expansion wave in the case of a thin plate shock wave interaction was larger than that in water surface interaction. The reflection coefficient calculated form the experimental results $R_{p \text {-exp }}$ is larger with thicker plate thickness in the plate case of PS and acrylic. The theoretically calculated reflection coefficient $R_{p \text {-theo }}$ is in rough agreement with $R_{p \text {-exp }}$.

The present paper reports experimental results of shock wave interaction with the interface made of simulated biomedical materials regarding to the effect of each acoustic impedance of the material and the thickness of a thin plate for a qualitative evaluation. Further experimental and theoretical work is needed for quantitative evaluation of these phenomena.

\section{Acknowledgment}

This work was supported by a MEXT KAKENHI Grant No. 26420100.

\section{References}

Delius, M., Medical applications and bioeffects of extracorporeal shock waves, Shock Waves, Vol.4, (1994), pp.55-72.

Kedrinskii, V.K., Dynamic of the cavitation zone during an underwater explosion near a free surface, J. Appl. Mech. and Tech. Phys., Vol.16, No.5, (1975), pp.724-733.

Kedrinskii, V.K., Negative pressure profile in cavitation zone at underwater explosion near free surface, Acta Astronoutica, Vol.3, (1976), pp.623-632.

Nakagawa, A., Manley, G.T., Gean, A.D., Ohtani, K., Armonda, R., Tsukamoto, A., Yamamoto, H., Takayama, K., Tominaga, T., Mechanisms of primary blast-induced traumatic brain injury: Insights from shock-wave research, J. Neurotrauma, Vol.28, (2011), pp.1101-1119.

National Astronomical Observatory of Japan (ed.), Chronological Scientific Tables, Maruzen, Tokyo, (2015). (in Japanese)

Ohtani, K., Ogawa, T., Micro-explosive-induced underwater shock wave propagation and reflection at the interface, Sci. Tech. Energetic Materials, Vol.76, No.6, (2015), pp.139-143.

Ohtani, K., Hashimoto, T., Takayama, K., Expansion wave propagation and cavitation bubble generation near the water surface, Proc. 27th Int. Symp. Shock Waves (ISSW27), (2009), pp.297.

Ohtani, K., Ogawa, T., Obayashi, S., Expansion wave and bubble generation by underwater micro explosion, Proc. 8th Int. Conf. Flow Dynamics (ICFD2011), (2011), pp.134-135.

Raum, K., Brandt, J. Simultaneous determination of acoustic impedance, longitudinal and lateral wave velocities for the characterization of the elastic microstructure of cortical bone, Proc. World Congress Ultrasound, (2003), pp.321-324.

Takayama, K., Saito, T., Shock wave/ geophysical and medical applications, Annual Review of Fluid Mechanics, Vol.36, (2004), pp.347-379.

Tomita, Y., Obara, T., Takayama, K., Kuwahara, M., Cavitation phenomena in extracorporeal microexplosion lithotripsy, Shock Waves, Vol.3, (1994), pp.149-157. 